\title{
Intraoperative contrast-enhanced ultrasound for intramedullary spinal neoplasms: patient series
}

\author{
Ariana Barkley, MD, Lynn B. McGrath Jr., MD, and Christoph P. Hofstetter, MD, PhD \\ Department of Neurological Surgery, University of Washington, Seattle, Washington
}

\begin{abstract}
BACKGROUND Primary intramedullary spinal tumors cause significant morbidity and death. Intraoperative ultrasound as an adjunct for localization and monitoring the extent of resection has not been systematically evaluated in these patients; the effectiveness of intraoperative contrast-enhanced ultrasound (CEUS) remains almost completely unexplored.

OBSERVATIONS A retrospective case series of patients at a single institution who had consented to the off-label use of intraoperative CEUS was identified. Seven patients with a mean age of $52.8 \pm 15.8$ years underwent resection of intramedullary tumors assisted by CEUS performed by a single attending neurosurgeon. Histopathological evaluation revealed 3 cases of hemangioblastoma, 1 case of pilocytic astrocytoma, 2 cases of ependymoma, and 1 case of subependymoma. Contrast enhancement correlated with gadolinium enhancement on preoperative magnetic resonance imaging. Intraoperative CEUS facilitated precise lesion localization and myelotomy planning. Dynamic CEUS studies were useful in demonstrating the blood supply to lesions with a dominant vascular pedicle. Regardless of contrast uptake, the differential enhancement between spinal cord tissue and neoplasm assisted in determining interface boundaries.
\end{abstract}

LESSONS Intraoperative CEUS constitutes a useful adjunct for the intraoperative delineation of contrast-enhancing intramedullary tumors and in vivo confirmation of gross-total resection. Systematic investigation is needed to establish the role of CEUS for resection of intramedullary spinal tumors of various pathologies.

https://thejns.org/doi/abs/10.3171/CASE2083

KEYWORDS contrast-enhanced ultrasound; intramedullary spinal tumors; spinal tumors; imaging; case series; Definity; ultrasound

Primary intramedullary spinal cord tumors are rare and account for only $2 \%-4 \%$ of all primary central nervous system (CNS) tumors. ${ }^{1}$ However, left untreated, they significantly diminish functional status and quality of life and are associated with increased mortality. The 2 most significant preoperative variables that determine prognosis include baseline neurological status and tumor histology; the most common entities in adults are ependymoma, astrocytoma, or hemangioblastoma. ${ }^{2}$ Maximal gross-total resection (GTR) remains the standard of care for initial treatment; however, the ability to safely achieve this surgically depends heavily on the quality of tissue planes at the spine-tumor tissue interface, a characteristic that varies widely across pathologies.

Techniques to augment visualization of the brain-tumor tissue interface have been developed to facilitate GTR of primary brain tumors, including intraoperative magnetic resonance imaging (MRI), 5-aminolevulinic acid (5-ALA), fluorescein, and intraoperative ultrasound. Although these tools have demonstrated benefit in certain situations, obstacles continue to prevent their widespread adoption. ${ }^{3-9}$ Intraoperative MRI requires a large capital investment and prolongs operative time while subjecting the patient to serial undraping and redraping. ${ }^{10} \mathrm{MRI}$ also remains vulnerable to anatomical error secondary to physical perturbations such as cerebrospinal fluid drainage or resection that are not accounted for without new image acquisition. ${ }^{11,12}$ Fluorescence-guided resection agents such as 5-ALA and fluorescein demarcate tumor tissue only on the surface of the resection cavity and are associated with potential phototoxicity, and efficacy studies have largely been restricted to high-grade gliomas, limiting applicability to broader histopathology. ${ }^{11,13}$

Conversely, conventional two-dimensional B-mode ultrasound imaging has managed to preemptively overcome the limitations of

ABBREVIATIONS 5-ALA = 5-aminolevulinic acid; CEUS = contrast-enhanced ultrasound; CNS = central nervous system; GTR = gross-total resection; MRC = Medical Research Council; MRI = magnetic resonance imaging.

INCLUDE WHEN CITING Published February 15, 2021; DOI: 10.3171/CASE2083.

SUBMITTED October 28, 2020. ACCEPTED October 30, 2020.

(C) 2021 The authors, CC BY-NC-ND 4.0 (http://creativecommons.org/licenses/by-nc-nd/4.0/). 
recent intraoperative technology by serving as a portable, economically accessible, in situ technical aid to characterize intraparenchymal pathology in real time. Few studies, however, have evaluated the use of intraoperative ultrasound for resection of intramedullary spinal cord tumors. ${ }^{14-18}$ Histopathological characteristics contribute to differences in the echogenicity of pathological versus normal tissue on B-mode ultrasound, with tumors such as low-grade gliomas exhibiting less obvious border distinctions. ${ }^{18}$ Intraoperative contrast-enhanced ultrasound (CEUS) has been proposed as a means of enhancing tumor boundaries to facilitate resection of brain tumors; however, many of these proposals provide a limited pathological scope. ${ }^{19-22}$ Here we present a case series evaluating the intraoperative utility of CEUS in visualizing intramedullary spinal cord tumors with various preoperative MRI characteristics.

\section{Study Description}

This study is a retrospective case series that was performed in accord with the rules and regulations of the University of Washington Institutional Review Board. All patients consented to the use of off-label intraoperative CEUS. All patients underwent preoperative MRI of the entire neuroaxis for surgical planning and to rule out other clinically significant areas of disease. Microsurgical tumor resection was performed by a single attending neurosurgeon (C.P.H.). Intraoperative neurophysiological monitoring was performed in all patients and included somatosensory evoked potentials, motor evoked potentials, and epidural $D$ waves.

For the surgical procedure, a standard laminectomy was performed at the index level, confirmed with fluoroscopic guidance. Prior to the durotomy, a premium level ultrasound system (EPIQ, Philips) with a linear probe (L12-5, Philips) was brought into the surgical field, draped in a surgical sterile plastic sheath along with $5 \mathrm{ml}$ of ultrasound transducing gel. The probe was placed over the dura to acquire standard B-mode imaging scans. The lesion was identified via sagittal images, and the laminectomy was expanded if necessary. Following the durotomy, a $10-\mu \mathrm{l} / \mathrm{kg}$ intravenous bolus of Definity was administered by our anesthesia team. Upon delivery of the contrast bolus, continuous CEUS was performed utilizing a low acoustic power, with continuous image acquisition of the bolus wash-in recorded. Following CEUS, maximal safe tumor microsurgical resection was performed with the assistance of an intraoperative microscope (Pentero, Zeiss). After tumor resection, an additional $10-\mu \mathrm{l} / \mathrm{kg}$ intravenous bolus of Definity was administered and intraoperative CEUS was repeated to assess for possible residual tumor.

\section{Discussion \\ Observations}

Seven patients with a mean age of $52.8 \pm 15.8$ years consented to undergo intraoperative CEUS to assist with tumor resection. Intramedullary tumors were located in either the cervical or thoracic spinal cord. Four cases ( 3 hemangioblastomas and 1 ependymoma) displayed vivid contrast enhancement on preoperative MRI, which was mirrored on intraoperative CEUS. CEUS appeared to serve as a useful intraoperative adjunct to confirm GTR in tumors with contrast enhancement. Here we present 1 case example for each pathology, highlighting pathology-specific CEUS features. A summary of all cases is shown in Table 1.

\section{Case 1}

Presentation. A 69-year-old man presented with 3 years of progressively worsening left greater than right upper-extremity dexterity, gait instability, and left lower-extremity weakness requiring a walker for ambulatory assistance. Neurological examination was notable for a Medical Research Council (MRC) motor score of $4 / 5$ in the left deltoid, gait ataxia, and a right-sided positive Babinski sign. MRI demonstrated a contrast-enhancing cervical intramedullary spinal cord tumor without an exophytic component and extensive spinal cord edema (Fig. $1 \mathrm{~A}$ and $\mathrm{C}$ ).

TABLE 1. Intraoperative CEUS case series

\begin{tabular}{|c|c|c|c|c|c|c|c|c|}
\hline $\begin{array}{l}\text { Age } \\
\text { (yrs) }\end{array}$ & Sex & Presentation & $\begin{array}{l}\text { Path } \\
\text { Location }\end{array}$ & MRI Signal & $\begin{array}{l}\text { Intraop } \\
\text { Ultrasound } \\
\text { Standard } \\
\text { B-Mode }\end{array}$ & Intraop CEUS & Pathology & $\begin{array}{l}\text { Extent of } \\
\text { Resection }\end{array}$ \\
\hline 69 & M & $\begin{array}{l}\text { Bilat arm weakness, ataxia, } \\
\text { myelopathy }\end{array}$ & Cervical & $\begin{array}{l}\text { T1 iso, T2 hyper, } \\
\text { enhancing }\end{array}$ & Hyperechoic & Enhancement & Hemangioblastoma & GTR \\
\hline 65 & $\mathrm{~F}$ & $\begin{array}{l}\text { Rt arm paresthesia, ataxia, } \\
\text { myelopathy }\end{array}$ & $\begin{array}{l}\text { High } \\
\text { thoracic }\end{array}$ & $\begin{array}{l}\text { T1 iso, T2 hyper, } \\
\text { enhancing }\end{array}$ & Isoechoic & Enhancement & Hemangioblastoma & $\begin{array}{l}\text { STR/no } \\
\text { adjunctive } \\
\text { treatment }\end{array}$ \\
\hline 47 & M & $\begin{array}{l}\text { Bilat leg paresthesia, myelopathy, } \\
\text { weakness }\end{array}$ & $\begin{array}{l}\text { High } \\
\text { thoracic }\end{array}$ & $\begin{array}{l}\text { T1 iso, T2 iso, } \\
\text { enhancing }\end{array}$ & Isoechoic & Enhancement & Hemangioblastoma & GTR \\
\hline 26 & M & $\begin{array}{l}\text { Lt leg paresthesia, weakness; } \\
\text { bowel/bladder dysfunction }\end{array}$ & $\begin{array}{l}\text { Low } \\
\text { thoracic }\end{array}$ & $\begin{array}{l}\mathrm{T} 1 \text { iso, } \mathrm{T} 2 \text { iso, } \\
\text { nonenhancing }\end{array}$ & Isoechoic & $\begin{array}{c}\text { No } \\
\text { enhancement }\end{array}$ & $\begin{array}{c}\text { Pilocytic } \\
\text { astrocytoma }\end{array}$ & GTR \\
\hline 59 & $F$ & $\begin{array}{l}\text { Bilat arm paresthesia and } \\
\text { myelopathy }\end{array}$ & Cervical & $\begin{array}{l}\text { T1 iso, T2 hyper, } \\
\text { enhancing }\end{array}$ & Isoechoic & Enhancement & Ependymoma & GTR \\
\hline 40 & M & $\begin{array}{l}\text { Rt leg paresthesia, weakness; } \\
\text { bowel/bladder dysfunction }\end{array}$ & $\begin{array}{l}\text { Low } \\
\text { thoracic }\end{array}$ & $\begin{array}{l}\text { T1 iso, T2 hypo, } \\
\text { nonenhancing }\end{array}$ & Isoechoic & $\begin{array}{c}\text { No } \\
\text { enhancement }\end{array}$ & Subependymoma & STR \\
\hline
\end{tabular}

hyper = hyperintense; iso = isointense; STR = subtotal resection. 


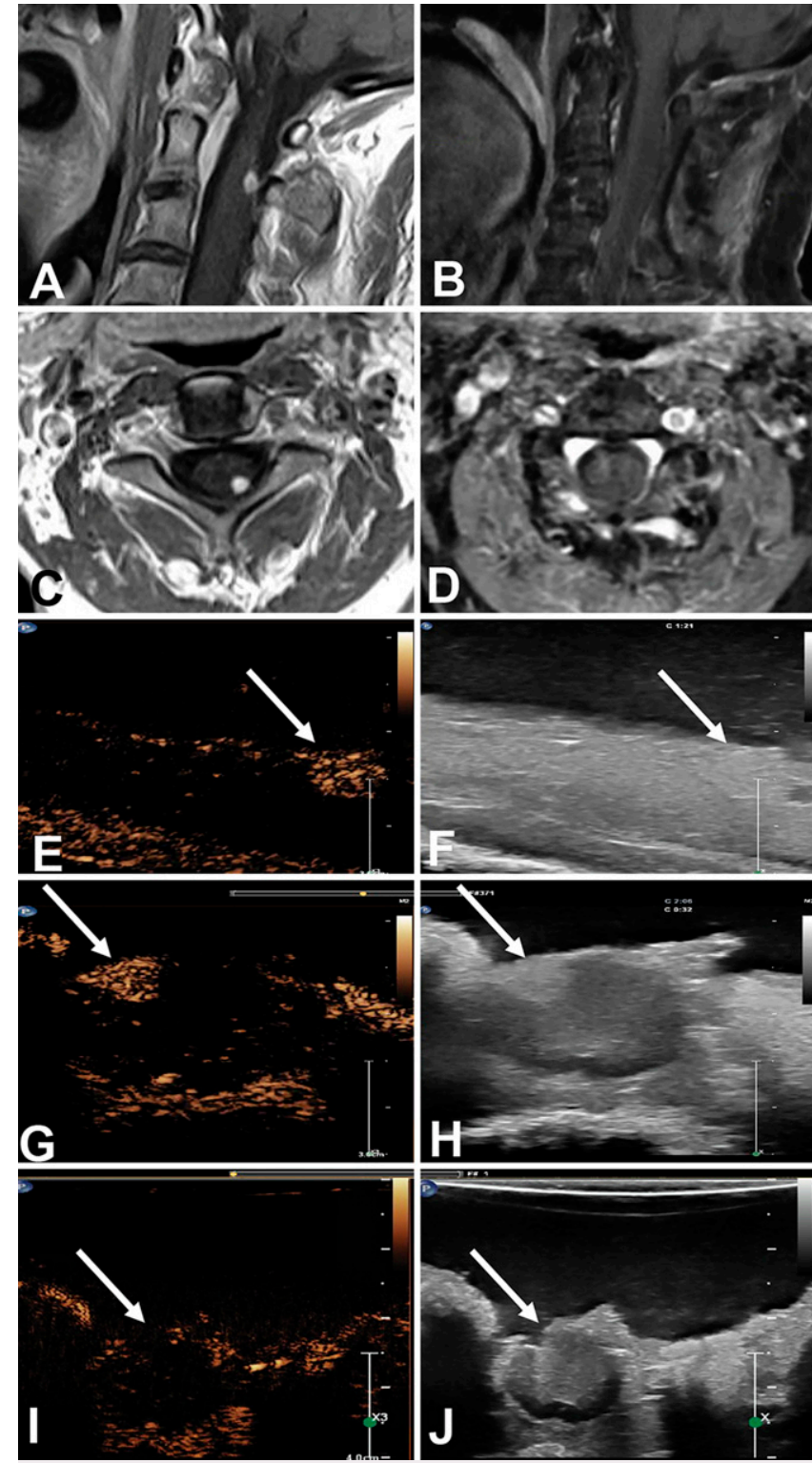

FIG. 1. Preoperative (A) and postoperative (B) sagittal T1 postcontrast. Preoperative (C) and postoperative (D) axial T1 postcontrast. Preresection postcontrast $(E)$ and $B$-mode $(F)$ sagittal images of the cervical lesion (arrows). Preresection postcontrast $(\mathbf{G})$ and B-mode $(\mathbf{H})$ axial images of the cervical lesion (arrows). Postresection postcontrast (I) and B-mode (J) axial images of resection cavity (arrows).

Intraoperative Ultrasound and Histopathological Findings. The patient underwent a C2-3 laminectomy with neurophysiological monitoring for resection of the cervical mass. Transdural sagittal B-mode insonation was performed to confirm that our exposure permitted sufficient access to the lesion. This demonstrated a moderately well-demarcated intramedullary mass on sagittal and axial images. Definity was administered per protocol, and the tumor exhibited avid contrast uptake in sharp contrast to the surrounding spinal cord (Fig. 1E-H). Intraoperative CEUS also demonstrated the location of the primary vascular supply in the pial plane prior to beginning resection, which permitted early coagulation and aided in minimizing hemorrhage during resection. Definity was again administered after resection was completed and CEUS images were obtained, demonstrating the absence of any contrast enhancement in the resection cavity (Fig. 1I and J). Histopathology was consistent with hemangioblastoma. The patient tolerated the procedure well, and postoperative MRI suggested a GTR of the lesion.

Postoperative Follow-Up. At his 3-month follow-up, the patient reported improvement in his preoperative symptoms and no longer required a walker for ambulation.

\section{Case 2}

Presentation. A 59-year-old woman presented with progressive upper-extremity numbness, weakness, and gait instability. Neurological examination was notable for reduced sensation in the bilateral 4th and 5th digits, as well as an abnormal left-sided Babinski reflex. MRI demonstrated a large intramedullary spinal cord tumor spanning C5-6 that was avidly contrast enhancing and associated with significant edema (Fig. 2A and C).

Intraoperative Ultrasound and Histopathological Findings. The patient underwent a C4-7 laminectomy with neurophysiological monitoring for resection of her intramedullary mass. Definity was administered per protocol, and intraoperative CEUS again clearly demarcated the intramedullary tumor from the surrounding spinal cord tissue. Importantly, CEUS also demonstrated a ventral vascular pedicle originating from the anterior spinal artery (Fig. 2E-J). This greatly facilitated resection of the tumor tissue because it allowed for swift centripetal dissection of tumor tissue from the ventral spinal cord tissue and meticulous dissection of the vascular pedicle. An additional bolus of Definity was administered after resection that demonstrated the clear absence of contrast uptake indicative of GTR, which was confirmed on postoperative MRI (Fig. 2B, D, I, and J). Histopathology was consistent with ependymoma, World Health Organization grade II. The patient tolerated the procedure well, with only mild deficits in lower-extremity proprioception and an uneventful recovery.

Postoperative Follow-up. At 2-weeks' follow-up, the patient reported that her paresthesias and gait instability had significantly improved since surgery.

\section{Case 3}

Presentation. A 26-year-old man presented with 2 years of progressive left lower-extremity paresthesias, 3 months of lower-extremity weakness requiring a cane for ambulation, and bowel and bladder dysfunction in conjunction with severe intermittent lower back pain extending to the S1-3 dermatomes. Neurological examination was notable for an MRC motor score of 4/5 in left knee extension, dorsiflexion, plantar flexion, and in the extensor hallucis longus. MRI was notable for a T11-12 intradural intramedullary T1 and T2 isoechoic mass with minimal contrast enhancement (Fig. 3A-B).

Intraoperative Ultrasound and Histopathological Findings. The patient underwent a T11-12 laminectomy with neurophysiological monitoring for resection. Transdural insonation was performed and revealed an isoechoic conus medullaris mass with a subtle tissue plane between neoplastic and normal tissue (Fig. 3C-F). Definity was administered per protocol but did not demonstrate contrast enhancement of the intramedullary lesion. However, given the physiological uptake of contrast by the surrounding spinal cord tissue, the lesion was able to be delineated (Fig. 3C-F). Microdissection was utilized to excise the tumor after sacrifice of four dorsal sacral nerve roots without neurophysiological abnormality on monitoring. The tumor demonstrated a tissue 

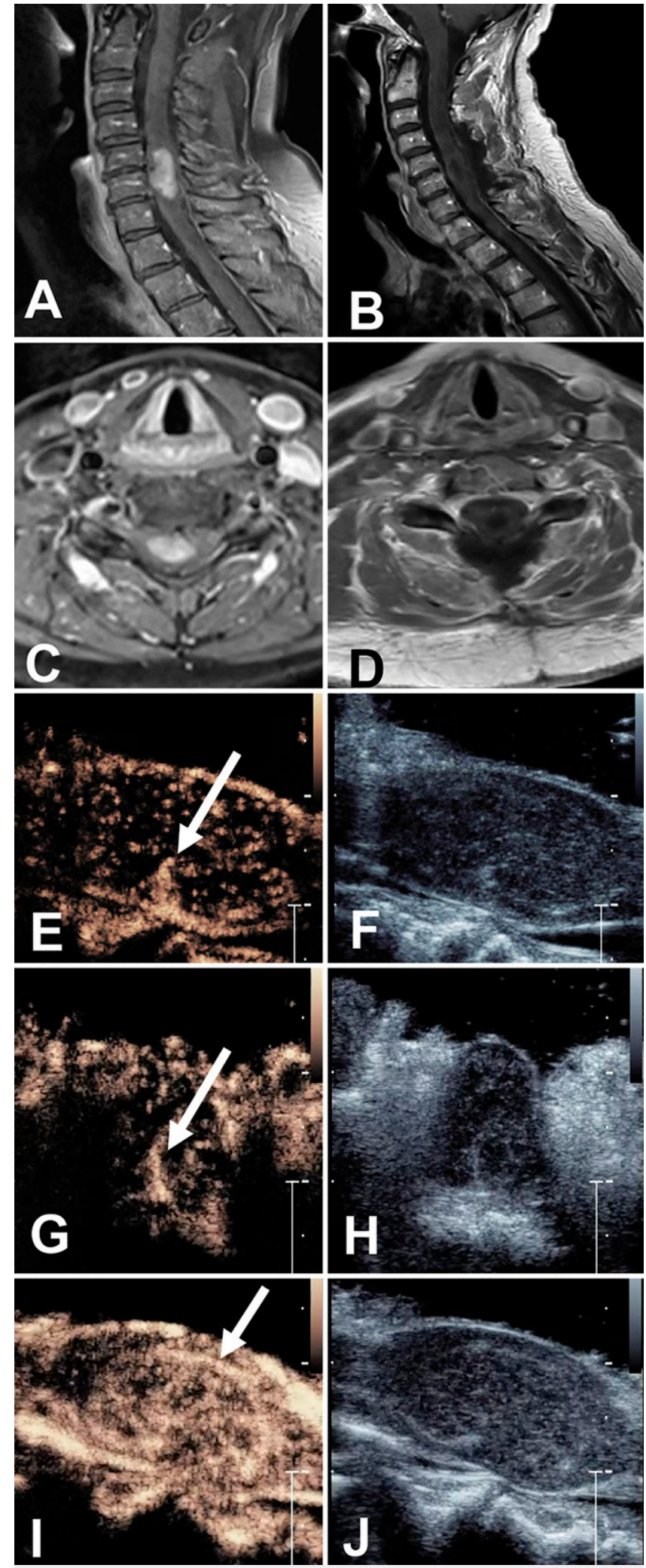

FIG. 2. Preoperative (A) and postoperative (B) sagittal T1 postcontrast. Preoperative (C) and postoperative (D) axial T1 postcontrast. Preresection postcontrast $(\mathbf{E})$ and B-mode $(\mathbf{F})$ sagittal images of a cervical lesion demonstrating a ventral vascular pedicle (arrow).

FIG. 2. (continued) $\rightarrow$
FIG. 2. Preresection postcontrast $(\mathbf{G})$ and $B$-mode $(\mathbf{H})$ axial images of a cervical lesion demonstrating a ventral vascular pedicle (arrow). Preresection precontrast (I) and B-mode (J) sagittal images demonstrating contrast uptake in mass, delineation of interface with normal tissue (arrow).

plane amenable to en bloc excision via an approach through the dorsal root entry zone. Histopathological diagnosis was confirmed as pilocytic astrocytoma of the conus medullaris.

Postoperative Follow-Up. In the immediate postoperative period, the patient's paresthesias and weakness improved, returning to full strength with only allodynia in the right second toe, which improved prior to discharge home.

\section{Lessons}

In this case series we demonstrate that intraoperative CEUS can serve as a useful adjunct to intraoperative visualization during resection of intramedullary spinal cord tumors. A recent study by Han et al. reported the use of intraoperative CEUS in 14 patients with intramedullary spine pathology. ${ }^{22}$ Despite these initial efforts, intraoperative ultrasound characteristics of various pathologies have yet to be catalogued. Our case series offers insights into preoperative imaging characteristics that may predict intraoperative visibility of lesions with this method. Our technique also demonstrates that intraoperative CEUS can be used for visualization of a vascular pedicle when present, thus facilitating resection. Finally, these results show that intraoperative CEUS remains beneficial for localizing small lesions intraoperatively.

In our series, intramedullary tumors that demonstrated contrast enhancement on preoperative MRI reliably demonstrated uptake of ultrasonic contrast on evaluation with intraoperative CEUS. This pattern follows that of high-grade intracranial gliomas, as demonstrated in a study by Prada and colleagues ${ }^{4}$ in which MRI and CEUS enhancement were found to correspond in 9/10 cases. In 1 case, CEUS revealed enhancement of the entire tumor bulk, whereas gadoliniumenhanced T1-weighted MRI demonstrated peripheral contrast enhancement. These differences may be attributable to the different properties of the contrast agents used for MRI compared to those used with CEUS. MRI utilizes gadolinium, which, after an initial vascular phase, accumulates in adjacent tumor tissue due to blood-brain barrier disruption. CEUS utilizes microbubbles, which remain within the vessels and therefore strictly reflect the vascular network of the lesion. The correlation between tumor vascularity and ultrasonic contrast enhancement is well established in various organ systems. ${ }^{16,23-25}$ Cheng and colleagues report a significant correlation in intracranial gliomas between the absolute CEUS peak intensity and the tumor microvessel density. ${ }^{19}$ In our series, hemangioblastomas demonstrated the most avid contrast enhancement. This is in keeping with the vascular nature of these lesions, which consist of fenestrated vascular channels lined by endothelial cells. ${ }^{26}$ Increased contrast uptake was also observed in the ependymoma, whereas no contrast uptake was noted in a case of a pilocytic astrocytoma or subependymoma. These findings may suggest that preoperative contrast enhancement may intraoperatively predict hyperechogenicity of the intramedullary lesion.

In our series, intraoperative CEUS assisted with identification of the vascular pedicle in highly vascular lesions. Likewise, Prada and colleagues benefited from early recognition of the arterial supply and venous drainage using intraoperative CEUS for resection of malignant brain gliomas. ${ }^{4}$ Knowledge of the precise location of the vascular pedicle is helpful to plan an efficient resection strategy based on the 


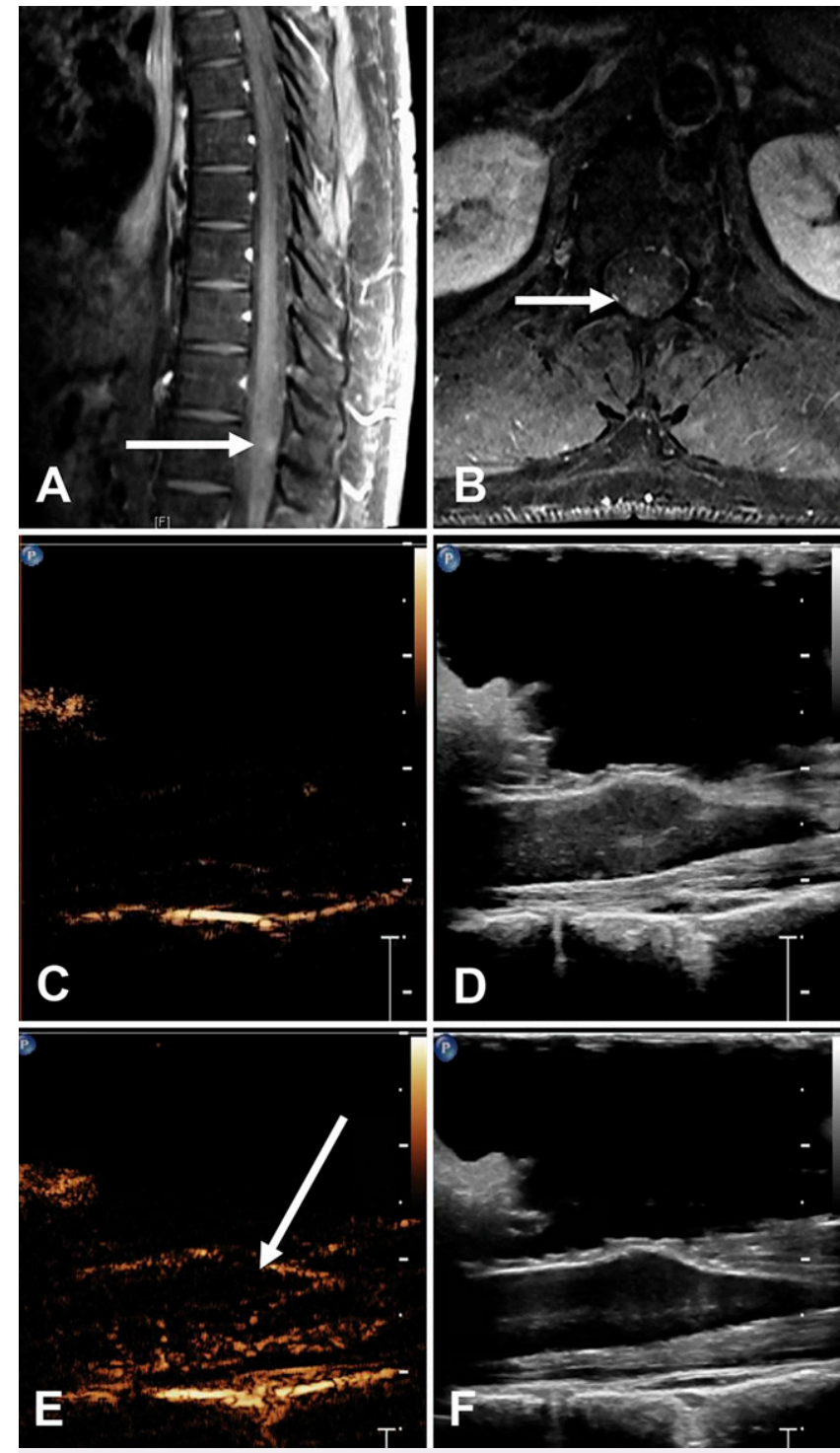

FIG. 3. Preoperative sagittal (A) and axial (B) T1 postcontrast thoracic lesion (arrows). Preresection precontrast (C) and B-mode (D) sagittal images of a thoracic lesion. Preresection postcontrast (E) and $B$-mode $(F)$ sagittal images of a thoracic lesion demonstrating minimal contrast enhancement (arrow).

location of the vascular supply. In all 3 cases with hemangioblastoma, superficial vascular pedicles within the pial plane were seen, facilitating early coagulation and ligation of the arterial supply. Similarly, Han et al. ${ }^{22}$ noted this ability to delineate afferent vascular channels, facilitating safe resection.

Our technique for utilizing intraoperative CEUS in spinal intramedullary tumor resection can assist with identifying lesions that would otherwise be difficult to localize prior to durotomy given the dearth of intraoperative intramedullary spinal cord navigation options. Our method was modified from one initially popularized by Maiuri et al., who divided the use of intraoperative ultrasound for intradural spinal cord lesions into 3 steps: the first scanning occurs after laminectomy prior to durotomy, the second after durotomy with scanning on the spinal cord itself, and the third after resection to facilitate identification of residual pathology. ${ }^{27}$ Like Han and colleagues, we also utilized this sequence with the adjustment of administering ultrasound contrast for identification through scanning prior to durotomy. ${ }^{22}$ Importantly, we found the contrast uptake differential between lesion and spinal cord tissue helpful for delineation of the tumor borders, even in those tumors that did not enhance during the resection. Therefore, regardless of predictions that can be made regarding hyperechogenicity through preoperative imaging, it should be considered as an intraoperative means of localizing lesions that is particularly advantageous for both small pathologies and large neoplasms that span multiple levels. Furthermore, we found using intraoperative CEUS after initial resection in contrast-enhancing lesions was useful in detecting residual tumor, thereby informing further surgical excision.

Despite early papers demonstrating the utility of intraoperative ultrasound and rates of GTR comparable with those using intraoperative MRI, ${ }^{11,13,20,28-32}$ a recent Cochrane review evaluating intraoperative adjuncts for CNS tumor resection found no currently ongoing prospective studies exploring intraoperative ultrasound..$^{33}$ The unique capabilities and economic advantages of intraoperative CEUS suggest that the technique merits further exploration as an adjunct in the resection of primary intramedullary spinal cord tumors. The technique also has implications outside of intramedullary spinal cord lesion resection given the ability to demonstrate subtle derangements in the spinal vasculature. This has made CEUS useful in the evaluation of hemodynamic perturbations from pathologies like spinal vascular malformations ${ }^{34,35}$ or spinal cord injury. ${ }^{36,37}$ In the future, it is therefore likely that CEUS can serve as a useful intraoperative and prognostic tool for a wide array of spinal cord pathology.

The current study has several limitations. It includes a small number of patients. Systematic research regarding CEUS characteristics of a wider range of CNS tumors will be necessary to adequately determine its role as a useful adjunct for resection of intramedullary spinal tumors. Preliminary case reports by Vetrano et al., ${ }^{21}$ Han et al., ${ }^{22}$ and our case series suggest that intraoperative CEUS is useful for the assessment of location, borders, and vascularity of intramedullary tumors. In cases of enhancing tumors, CEUS may also help to confirm completeness of initial resection.

In summary, intraoperative CEUS constitutes a safe and useful adjunct for resection of intramedullary spinal cord tumors. Preoperative imaging characteristics may predict echogenic differences compared with normal tissue. Further studies are needed to evaluate the potential role of CEUS for resection of intramedullary spinal tumors of various pathologies.

\section{Acknowledgments}

We gratefully acknowledge generous support from the Washington State Spinal Cord Injury Consortium (WASCIC), Craig H. Neilsen Foundation, United States Department of Defense, and James D. and Sherry Raisbeck Foundation.

We thank Shaun Bornemeier for excellent technical support of the intraoperative ultrasound system and Sharon Durfy, $\mathrm{PhD}$, for assistance with manuscript preparation.

\section{References}

1. Tobin MK, Geraghty JR, Engelhard HH, et al. Intramedullary spinal cord tumors: a review of current and future treatment strategies. Neurosurg Focus. 2015;39(2):E14.

2. Samartzis D, Gillis CC, Shih $P$, et al. Intramedullary spinal cord tumors: part i-epidemiology, pathophysiology, and diagnosis. Global Spine J. 2015;5(5):425-435. 
3. Hammoud MA, Ligon BL, elSouki R, et al. Use of intraoperative ultrasound for localizing tumors and determining the extent of resection: a comparative study with magnetic resonance imaging. J Neurosurg. 1996;84(5):737-741.

4. Prada F, Vitale V, Del Bene M, et al. Contrast-enhanced MR imaging versus contrast-enhanced US: a comparison in glioblastoma surgery by using intraoperative fusion imaging. Radiology. 2017;285(1):242-249

5. Liang D, Schulder M. The role of intraoperative magnetic resonance imaging in glioma surgery. Surg Neurol Int. 2012;3(4)(suppl 4):S320-S327.

6. Stummer W, Pichlmeier U, Meinel T, et al. Fluorescence-guided surgery with 5-aminolevulinic acid for resection of malignant glioma: a randomised controlled multicentre phase III trial. Lancet Oncol. 2006;7(5):392-401.

7. Garzon-Muvdi T, Kut C, Li X, et al. Intraoperative imaging techniques for glioma surgery. Future Oncol. 2017;13(19): 1731-1745.

8. Koc K, Anik I, Cabuk B, et al. Fluorescein sodium-guided surgery in glioblastoma multiforme: a prospective evaluation. $\mathrm{Br} J$ Neurosurg. 2008;22(1):99-103.

9. Neira JA, Ung TH, Sims JS, et al. Aggressive resection at the infiltrative margins of glioblastoma facilitated by intraoperative fluorescein guidance. J Neurosurg. 2017;127(1):111-122.

10. Ahmadi R, Campos B, Haux D, et al. Assessing perioperative complications associated with use of intraoperative magnetic resonance imaging during glioma surgery - a single centre experience with 516 cases. Br J Neurosurg. 2016;30(4):397-400.

11. Mahboob S, McPhillips R, Qiu Z, et al. Intraoperative ultrasoundguided resection of gliomas: a meta-analysis and review of the literature. World Neurosurg. 2016;92:255-263.

12. Bayer $S$, Maier $A$, Ostermeier $M$, et al. Intraoperative imaging modalities and compensation for brain shift in tumor resection surgery. Int J Biomed Imaging. 2017;2017:6028645

13. Eljamel MS, Mahboob SO. The effectiveness and costeffectiveness of intraoperative imaging in high-grade glioma resection; a comparative review of intraoperative ALA, fluorescein, ultrasound and MRI. Photodiagn Photodyn Ther. 2016;16:35-43.

14. Haciyakupoglu E, Yuvruk E, Onen MR, et al. The use of intraoperative ultrasonography in intradural spinal tumor surgery. Turk Neurosurg. 2019;29(2):237-241.

15. Harel R, Knoller N. Intraoperative spine ultrasound: application and benefits. Eur Spine J. 2016;25(3):865-869.

16. Prada F, Perin A, Martegani A, et al. Intraoperative contrastenhanced ultrasound for brain tumor surgery. Neurosurgery. 2014; 74(5):542-552.

17. Regelsberger J, Fritzsche E, Langer N, et al. Intraoperative sonography of intra- and extramedullary tumors. Ultrasound Med Biol. 2005;31(5):593-598.

18. Zhou H, Miller D, Schulte DM, et al. Intraoperative ultrasound assistance in treatment of intradural spinal tumours. Clin Neurol Neurosurg. 2011;113(7):531-537.

19. Cheng L-G, He W, Zhang $\mathrm{H}-\mathrm{X}$, et al. Intraoperative contrast enhanced ultrasound evaluates the grade of glioma. BioMed Res Int. 2016;2016:2643862.

20. He W, Jiang $X Q$, Wang $S$, et al. Intraoperative contrast-enhanced ultrasound for brain tumors. Clin Imaging. 2008;32(6):419-424.

21. Vetrano IG, Prada F, Nataloni IF, et al. Discrete or diffuse intramedullary tumor? Contrast-enhanced intraoperative ultrasound in a case of intramedullary cervicothoracic hemangioblastomas mimicking a diffuse infiltrative glioma: technical note and case report. Neurosurg Focus. 2015;39(2):E17.

22. Han B, Wu D, Jia W, et al. Intraoperative ultrasound and contrastenhanced ultrasound in surgical treatment of intramedullary spinal tumors. World Neurosurg. 2020;137:e570-e576.
23. Vitali F, Pfeifer L, Janson C, et al. Quantitative perfusion analysis in pancreatic contrast enhanced ultrasound (DCE-US): a promising tool for the differentiation between autoimmune pancreatitis and pancreatic cancer. Z Gastroenterol. 2015;53(10):1175-1181.

24. Sridharan R, Yunos SM, Aziz S, et al. Comparison on the use of semi-automated and automated core biopsy needle in ultrasound guided breast biopsy. Med J Malaysia. 2015;70(6):326-333.

25. Chaudhari MH, Forsberg F, Voodarla A, et al. Breast tumor vascularity identified by contrast enhanced ultrasound and pathology: initial results. Ultrasonics. 2000;38(1-8):105-109.

26. Chaudhry AP, Montes M, Cohn GA. Ultrastructure of cerebellar hemangioblastoma. Cancer. 1978;42(4):1834-1850.

27. Maiuri $F$, laconetta $G$, de Divitiis $O$. The role of intraoperative sonography in reducing invasiveness during surgery for spinal tumors. Minim Invasive Neurosurg. 1997;40(1):8-12.

28. Bal J, Camp SJ, Nandi D. The use of ultrasound in intracranial tumor surgery. Acta Neurochir (Wien). 2016;158(6):1179-1185.

29. Moiyadi A, Shetty P. Objective assessment of utility of intraoperative ultrasound in resection of central nervous system tumors: a cost-effective tool for intraoperative navigation in neurosurgery. J Neurosci Rural Pract. 2011;2(1):4-11.

30. Moiyadi AV, Shetty P, Degaonkar A. Resection of pediatric brain tumors: intraoperative ultrasound revisited. J Pediatr Neurosci. 2017;12(1):19-23.

31. Sastry R, Bi WL, Pieper S, et al. Applications of ultrasound in the resection of brain tumors. J Neuroimaging. 2017;27(1):5-15.

32. Mursch K, Scholz M, Brück W, et al. The value of intraoperative ultrasonography during the resection of relapsed irradiated malignant gliomas in the brain. Ultrasonography. 2017;36(1):60-65.

33. Jenkinson MD, Barone DG, Bryant A, et al. Intraoperative imaging technology to maximise extent of resection for glioma. Cochrane Database Syst Rev. 2018;1(1):CD012788.

34. Prada F, Del Bene M, Farago G, et al. Spinal dural arteriovenous fistula: is there a role for intraoperative contrast-enhanced ultrasound? World Neurosurg. 2017;100:712.e15-712.e18.

35. Della Pepa GM, Sabatino G, Sturiale CL, et al. Integration of realtime intraoperative contrast-enhanced ultrasound and color doppler ultrasound in the surgical treatment of spinal cord dural arteriovenous fistulas. World Neurosurg. 2018;112:138-142.

36. Khaing ZZ, Cates LN, DeWees DM, et al. Contrast-enhanced ultrasound to visualize hemodynamic changes after rodent spinal cord injury. J Neurosurg Spine. 2018;29(3):306-313.

37. Huang L, Chen K, Chen FC, et al. Intraoperative contrastenhanced ultrasonography for microcirculatory evaluation in rhesus monkey with spinal cord injury. Oncotarget. 2017;8(25): 40756-40764.

\section{Disclosures}

Dr. Hofstetter reports teaching and consulting activities for Joimax and Globus Medical.

\section{Author Contributions}

Conception and design: Hofstetter, McGrath. Acquisition of data: all authors. Analysis and interpretation of data: Barkley, McGrath. Drafting the article: Barkley, McGrath. Critically revising the article: Barkley, McGrath. Reviewed submitted version of manuscript: Barkley. Approved the final version of the manuscript on behalf of all authors: Hofstetter. Study supervision: Hofstetter.

\section{Correspondence}

Christoph P. Hofstetter: University of Washington, Seattle, WA. respub@uw.edu. 